\title{
Effects of cromakalim (BRL 34915) on potassium conductances in CA3 neurons of the guinea-pig hippocampus in vitro
}

\author{
C. Alzheimer, B. Sutor, and G. ten Bruggencate \\ Physiologisches Institut der Universität, Pettenkoferstrasse 12, D-8000 München 2, Federal Republic of Germany
}

\begin{abstract}
Summary. The action of the potassium channel activator, cromakalim (BRL 34915), on membrane potential, input resistance and current-voltage-relationship of $\mathrm{CA} 3$ neurons in a slice preparation of the guinea-pig hippocampus was investigated by means of intracellular recordings. In the presence of tetrodotoxin, cromakalim $(30-100 \mu \mathrm{mol} / \mathrm{l})$ produced a hyperpolarization up to $4 \mathrm{mV}$ associated with a decrease in input resistance up to $10 \mathrm{MOhms}$. Determination of the equilibrium potential of the cromakalim action revealed that the hyperpolarization is due to the activation of a potassium conductance. This cromakalim-activated potassium conductance was voltage-dependent, i.e. it increased with hyperpolarization. Among a number of potassium channel blockers tested, only $\mathrm{Cs}^{+}(2 \mathrm{mmol} / \mathrm{l})$ and $\mathrm{Ba}^{2+}$ $(0.5 \mathrm{mmol} / 1)$ were able to inhibit the cromakalim-induced effects. Simultaneously, both cations suppressed the hyperpolarizing inward rectification (anomalous rectification) in these neurons, indicating that cromakalim activated or potentiated an inwardly rectifying potassium conductance. In addition, cromakalim slightly enhanced both amplitude and duration of afterhyperpolarizations following single calcium-dependent action potentials, suggesting that cromakalim might have a weak facilitatory effect on calcium-dependent potassium conductances.
\end{abstract}

Key words: Hippocampal slice - CA3 - Cromakalim (BRL 34915) - Potassium conductances - Anomalous rectification

\section{Introduction}

The antihypertensive compound cromakalim (BRL 34915) has been shown to activate potassium channels in smooth muscle cells, thereby leading to a hyperpolarization of the membrane potential and to smooth muscle relaxation (Allen et al. 1986; Hamilton et al. 1986; Weir and Weston 1986; Coldwell and Howlett 1987; Nakao et al. 1988). With regard to the central nervous system, Alzheimer and ten Bruggencate (1988) demonstrated that in hippocampal slices cromakalim reduces epileptiform activity induced by changes in the ionic composition of the perfusion medium (elevation of $\mathrm{K}^{+}$, omission of $\mathrm{Ca}^{2+}$ or $\mathrm{Mg}^{2+}$ ). Also, at the same concentrations $(30-100 \mu \mathrm{M})$, cromakalim inhibited synaptic transmission as indicated by a reduction in the amplitude of the stimulus-evoked population spike. In ad-

Send offprint requests to $\mathrm{C}$. Alzheimer at the above address dition, intracellular recordings from $\mathrm{CA} 3$ neurons revealed that the compound decreased the frequency of intrinsically generated spontaneous burst discharges which occur in these neurons under normal conditions (see Hablitz and Johnston 1981). In particular, the latter observation led to the conclusion that cromakalim influences epileptiform activity by acting on intrinsic membrane conductances which control neuronal excitability (Alzheimer and ten Bruggencate 1988). The anticonvulsive activity of cromakalim has been confirmed in vivo by showing that the drug prevents epileptiform seizures induced by the mast cell degranulating peptide (Gandolfo et al. 1989).

The present study was designed to investigate in more detail the effects of cromakalim on intrinsic membrane properties of hippocampal $\mathrm{CA} 3$ pyramidal cells. Thus, the actions of cromakalim on membrane potential and current-voltagerelationships were studied in the presence of tetrodotoxin (TTX), i.e. in neurons functionally isolated from synaptic input. In addition, several potassium channel blockers were tested for their ability to abolish the effects of cromakalim.

\section{Methods}

Slices of the guinea-pig hippocampus were prepared and maintained as described previously (Alzheimer and ten Bruggencate 1988). The artificial cerebrospinal fluid (ACSF) contained (in mmol/1): $\mathrm{NaCl} 118, \mathrm{KCl} 3, \mathrm{CaCl}_{2} 1.5, \mathrm{MgCl}_{2}$ 1, $\mathrm{NaH}_{2} \mathrm{PO}_{4}$ 1.2, $\mathrm{NaHCO}_{3} 22$, D-glucose 11. The solution was gassed continuously with a mixture consisting of $95 \%$ $\mathrm{O}_{2}$ and $5 \% \mathrm{CO}_{2}$ in order to obtain a $\mathrm{pH}$ value of 7.4 at a temperature of $30-31^{\circ} \mathrm{C}$. When $\mathrm{CsCl}$ or tetraethylammonium chloride (TEA) were added to the ACSF, equimolar amounts of $\mathrm{NaCl}$ were omitted from the solution.

Intracellular recordings from CA3 pyramidal neurons were performed by means of glass microelectrodes filled with either $3 \mathrm{~mol} / \mathrm{l} \mathrm{KCl}$ or $2 \mathrm{~mol} / \mathrm{l} \mathrm{CsCl}$. The electrode resistance ranged between 35 and $80 \mathrm{MOhms}$. Intracellular signals were recorded and amplified using an Axoclamp 2 amplifier (Axon Instruments, Burlingame, CA, USA) or, in more recent experiments, an npi SEC 1L amplifier (npi, Tamm, FRG). For current passage via the recording electrode both devices employ the discontinuous current injection technique ("switched current clamp"). Using the npi SEC 1L amplifier, single-electrode voltage-clamp recordings were performed at switching frequencies of $18-22 \mathrm{kHz}(25 \%$ duty cycle). The adjustment of the amplifier and the criteria for the selection of microelectrodes have been described previously (Sutor and Zieglgänsberger 1987). Intracellular 
signals obtained in the course of current- and voltage-clamp measurements of $\mathrm{I} / \mathrm{V}$-relations were averaged on-line (4-6 sweeps) and stored using a laboratory computer system (CED 1401, Cambridge Instruments, Cambridge, UK, in conjunction with a Tandon PCA 30). The membrane potential and the input resistance $\left(R_{N}\right.$, determined by injection of current pulses of $0.2 \mathrm{nA}$ at a frequency of $0.1-0.2 \mathrm{~Hz}$ ) were continuously monitored on a chart recorder (Gould System Instruments, Cleveland, OH, USA) and stored on magnetic tape (TEAC MR30, Tokyo, Japan, frequency response of the tape recorder DC- $2.5 \mathrm{kHz}$ ) for off-line analysis. The burst frequency of CA3 neurons was determined using a spike rate analyser. Action potentials were photographed directly from the screen of an analog storage oscilloscope (Tektronix, Beaverton, OR, USA) and hard copies of the digitised data were obtained with a HP $7470 \mathrm{~A}$ plotter.

Cromakalim $[( \pm) 6$ - cyano-3,4 - dihydro-2,2-dimethyl trans-4-(2-oxo-1 - pyrrolidyl)-2H-benzo[b]pyran-3-ol] was kindly provided by Beecham-Wülfing (Gronau, FRG). Stock solutions of cromakalim $(30 \mathrm{mmol} / \mathrm{l})$ were made up freshly in dimethyl sulfoxide (DMSO) and diluted in ACSF to reach final concentrations between 30 and $100 \mu \mathrm{mol} / \mathrm{l}$. Final solvent concentrations were less than $0.33 \%$. At this concentration, DMSO had no significant effects when applied in control experiments $(n=4)$.

CA3 pyramidal neurons were "isolated" from synaptic input by adding $0.6 \mu \mathrm{mol} / 1 \mathrm{TTX}$ to the bathing solution. Potassium conductances were blocked by TEA $(10-$ $15 \mathrm{mmol} / \mathrm{l}$ ), 4-amino pyridine (4-AP, $0.1 \mathrm{mmol} / \mathrm{l}), \mathrm{BaCl}_{2}$ $(0.5 \mathrm{mmol} / 1)$, and $\mathrm{CsCl}(2 \mathrm{mmol} / \mathrm{l})$. In a few experiments, tolbutamide $(0.5-1 \mathrm{mmol} / \mathrm{l})$ was added to the ACSF in order to block putative ATP-sensitive potassium conductances. All drugs were obtained from Sigma (Taufkirchen, FRG). The substances were applied by addition to the bathing solution. The flow rate of the superfusion system was set to $4-6 \mathrm{ml} / \mathrm{min}$.

Values are given as mean \pm standard error of the mean (SEM). Statistical comparisons of mean values were performed using the paired Student's $t$-test.

\section{Results}

Intracellular recordings were obtained from 61 neurons. Under control conditions (i.e. in the absence of TTX), the cells were selected on the basis of their resting membrane potential (RMP $>-60 \mathrm{mV})$, their input resistance $\left(\mathrm{R}_{\mathrm{N}}\right.$ $>50 \mathrm{MOhms}$ ), and the overshoot of spontaneous or directly evoked action potentials $(>15 \mathrm{mV})$. In normal ACSF, the neurons usually displayed spontaneous activity consisting of either single action potentials or of small burst discharges, where two to four single spikes were present on top of a small depolarizing wave (see Johnston et al. 1980 and Hablitz and Johnston 1981). The frequency of spontaneous discharges ranged between 0.5 and $4 \mathrm{~Hz}$.

\section{Effects of cromakalim on $R M P$ and $R_{\mathrm{N}}$}

Within 5-8 min following superfusion with a TTX-containing medium, a complete cessation of spontaneous and synaptically evoked firing of CA3 neurons was observed. In the presence of TTX, addition of $100 \mu \mathrm{mol} / 1 \mathrm{cromakalim}$ to the bathing solution consistently produced a hyperpolarization of the RMP by about $4 \mathrm{mV}$ and a decrease in $R_{N}$ by

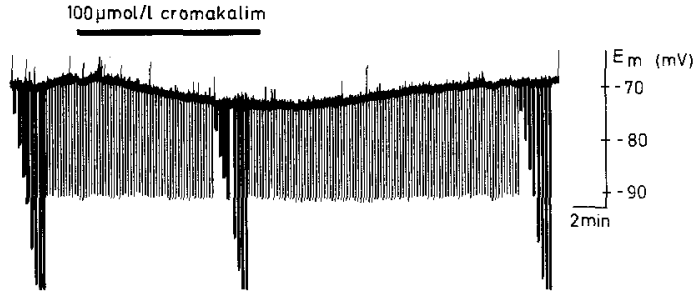

B
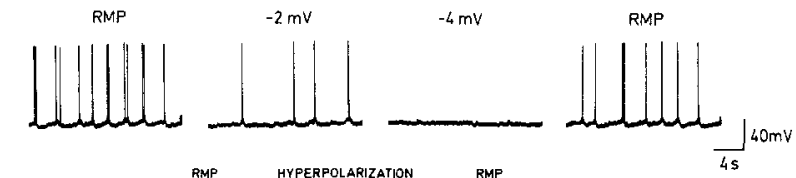

C

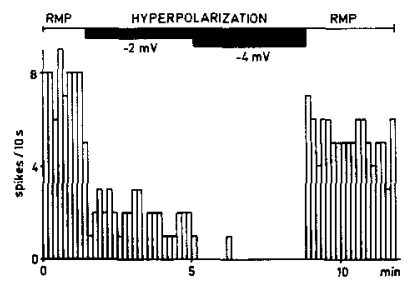

Fig. 1. Effects of cromakalim on RMP and $R_{N}$ of a TTX-treated hippocampal CA3 neuron in comparison to the effects of hyperpolarizing DC injection on the spontaneous activity of CA3 neurons. (A) Chart record of the membrane potential of a neuron in the absence and presence of $100 \mu \mathrm{mol} / 1 \mathrm{cromakalim}$ (application period indicated by the bar). Action potentials were blocked by the addition of $0.6 \mu \mathrm{mol} / \mathrm{ITT}$ to the bathing solution. The downward deflections represent voltage deviations produced by hyperpolarizing current pulses $(300 \mathrm{~ms}, 0.2 \mathrm{nA})$ injected at a frequency of $0.1 \mathrm{~Hz}$ in order to monitor $\mathrm{R}_{\mathrm{N}} \mathrm{I} / \mathrm{V}$-curves were determined before, during and after the application of cromakalim. In this neuron cromakalim hyperpolarized the membrane potential by $4 \mathrm{mV}$ and decreased the $\mathrm{R}_{\mathrm{N}}$ by $10 \mathrm{MOhms}$. (B) Representative recordings of spontaneous activity of a normal CA3 neuron. The membrane potential was $-65 \mathrm{mV}$ and the $\mathrm{R}_{\mathrm{N}} 100 \mathrm{MOhm}$. Injection of direct current with $0.02 \mathrm{nA}$ led to a hyperpolarization of $2 \mathrm{mV}$ associated with a decrease in the discharge rate. DC-injection of $0.04 \mathrm{nA}$ produced a hyperpolarization of $4 \mathrm{mV}$ and an abolition of spontaneous activity. Upon removal of the direct current, the spontaneous activity reappeared. (C) Ratemeter record from the same neuron as in B. Period of current injection is indicated by the bar

about 10 MOhms (Fig. 1 A, Table 1). Both, the cromakaliminduced hyperpolarization and the reduction in $R_{N}$ were reversible following washout of the drug (Fig. 1A, Table 1). Under the experimental conditions used, the hyperpolarization started 5-7 min after onset of drug application and reached its maximum after a further 10-15 min. Upon washout of cromakalim, RMP and $\mathrm{R}_{\mathrm{N}}$ recovered within $15-20 \mathrm{~min}$, a time course similar to that observed in extracellular recordings (Alzheimer and ten Bruggencate 1988). A paired $t$-test performed in 10 randomly selected cells revealed that the cromakalim-evoked changes in RMP and $R_{N}$ were highly significant (Table 1). In order to test whether such a small hyperpolarization would be sufficient to account for the inhibitory action of cromakalim observed in the absence of TTX, the firing rate of 3 representative CA3 neurons was determined at RMP $(-64 \mathrm{mV}$ to $-66 \mathrm{mV})$ and during hyperpolarizations by $2 \mathrm{mV}$ and $4 \mathrm{mV}$, respectively, using direct current (DC) injection (Fig. 1 B, C). The effects of such hyperpolarizations were comparable to the changes induced by $30 \mu \mathrm{mol} / 1$ and $100 \mu \mathrm{mol} / 1$ cromakalim, respec- 
Table 1. Effects of cromakalim on resting membrane potential and input resistance of CA3 pyramidal cells. All measurements $(\mathrm{N}=10$, $\overrightarrow{\mathrm{x}} \pm$ SEM) were obtained in TTX. For a statistical analysis of the effects of cromakalim (control vs. cromakalim), a paired $t$-test was used

\begin{tabular}{|c|c|c|c|c|}
\hline & \multicolumn{2}{|c|}{ Resting membrane potential } & \multicolumn{2}{|c|}{ Input resistance } \\
\hline & RMP (mV) & $\triangle \mathrm{RMP}(\mathrm{mV})$ & $\mathrm{R}_{\mathrm{N}}(\mathrm{M} \Omega)$ & $\Delta \mathrm{R}_{\mathrm{N}}(\mathrm{M} \Omega)$ \\
\hline
\end{tabular}

${ }^{*} p<0.0001 ;{ }^{* *} p<0.002$

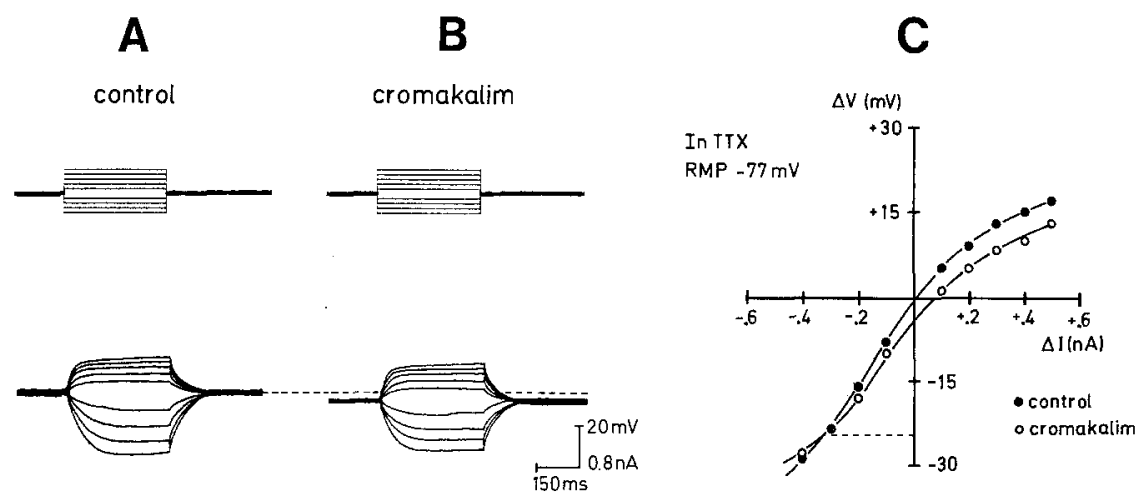

Fig. 2. Measurement of the $\mathrm{I} / \mathrm{V}$-relationship in the absence and presence of cromakalim and determination of the reversal potential of the action of cromakalim. (A) and (B) Determination of $\mathrm{I} / \mathrm{V}$-curves before (A) and during the application of cromakalim (100 $\mu \mathrm{mol} / 1, \mathbf{B})$ in the presence of $0.6 \mu \mathrm{mol} / \mathrm{l}$ TTX. De- and hyperpolarizing current pulses (upper traces) were injected and the corresponding voltage deviations (lower traces) measured at the end of the current pulse. The subsequent recordings are presented superimposed. The dashed line indicates the RMP $(-77 \mathrm{mV})$. (C) Plot of the voltage deviations as a function of the injected current in the absence (filled circles) and presence of cromakalim (open circles). Data from the neuron shown in $\mathbf{A}$ and $\mathbf{B}$. Both curves intercept at a potential value $25 \mathrm{mV}$ negative to the origin of the coordinate system. Since the origin corresponds to the RMP $(-77 \mathrm{mV})$, in this neuron the equilibrium potential of the action of cromakalim was $-102 \mathrm{mV}$

tively (see Alzheimer and ten Bruggencate 1988; Fig. 5). As shown by the ratemeter recording in Fig. $1 \mathrm{C}$, a hyperpolarization of the membrane potential by $4 \mathrm{mV}$ was sufficient to suppress the spontaneous activity in CA3 neurons (cf. Hablitz and Johnston 1981).

\section{Effects of cromakalim on the I/V-relation}

In order to study the cromakalim-induced conductance changes in more detail, I/V-relationships of CA3 pyramidal cells were determined by means of the switched currentclamp technique. Depolarizing and hyperpolarizing current pulses were injected and the corresponding voltage deviations were measured in the absence and presence of $100 \mu \mathrm{mol} / 1 \mathrm{cromakalim}$ (Fig. 2A, B). Under control conditions (i.e. in the presence of TTX), the I/V-curve was nonlinear in all neurons tested (Fig. 2C, closed circles). In the depolarizing direction, the slope resistance (as determined from the slope of the curve) decreased with increasing current strength (outward rectification), whereas in the hyperpolarizing direction the curve was almost linear in a potential range $15-20 \mathrm{mV}$ negative to the RMP. At current pulses with amplitudes larger than $-0.4 \mathrm{nA}$, the slope resistance again decreased (inward rectification). Following the application of cromakalim $(100 \mu \mathrm{mol} / \mathrm{l})$, a negative shift of the $\mathrm{I} / \mathrm{V}$-curve along the voltage-axis was observed, corresponding to the drug-induced hyperpolarization of the RMP (Fig. 2C, open circles). In addition, at every point of the curve there was a slight decrease in slope resistance which was clearly more pronounced in the hyperpolarizing direction as compared to the depolarizing direction. In order to quantify the difference, a rectification ratio ( $R R$, see Connors et al. 1982) was calculated from the input resistances obtained by injection of depolarizing and hyperpolarizing current pulses $(0.4 \mathrm{nA})$, respectively. Under control conditions, the RR was found to be $0.55 \pm 0.12(n=$ 4 ), indicating outward rectification. In the same neurons, cromakalim produced a significant increase in the RR to $0.67 \pm 0.12$ (paired $t$-test, $p<0.005$ ). In all cases, the increase in $R R$ was due to a significant decrease in the voltage changes produced by the hyperpolarizing current pulses, whereas voltage changes induced by the depolarizing current pulses remained constant or were only slightly decreased. This suggests that the decrease in the RR was the consequence of the activation of an inwardly rectifying conductance rather than the result of an inactivation of an outward rectifier.

In the hyperpolarizing direction, the I/V-curve obtained in the presence of cromakalim crossed the control curve in a potential range $20-30 \mathrm{mV}$ negative to the RMP (Fig. 2C). According to Ginsborg (1967), this interception point indicates the reversal potential of the cromakalim-induced hyperpolarization; measurements in 6 neurons revealed a mean value of the cromakalim equilibrium potential of $-100.3 \pm 3.8 \mathrm{mV}$. This value is similar to the Nernstian equilibrium potential for potassium ions under the exper- 
imental conditions used (assuming an intracellular potassium concentration of $120-150 \mathrm{mM}$, the $\mathrm{E}_{\mathrm{K}}$ ranged between $-96 \mathrm{mV}$ and $-102 \mathrm{mV}$ ), suggesting that the cromakalim-induced hyperpolarization was due to the activation of a potassium conductance. To confirm this, cromakalim was applied at RMP $(-67 \pm 2 \mathrm{mV}, n=4)$ and at a membrane potential of $-100 \mathrm{mV}$ obtained by hyperpolarizing DC injection. At RMP, cromakalim produced a mean hyperpolarization of $4.2 \pm 0.3 \mathrm{mV}$, whereas at $-100 \mathrm{mV}$, the cromakalim-evoked hyperpolarization was found to be abolished or reversed (mean value: $+0.25 \pm 0.4 \mathrm{mV}$ ), indicating a reversal potential close to $-100 \mathrm{mV}$.

The results obtained under current clamp conditions suggested that cromakalim hyperpolarizes hippocampal CA3 neurons by activation of a voltage-dependent, inwardly rectifying potassium conductance. The possible voltage-dependence of the cromakalim-activated conductance was further investigated by determining the V/I-relationship of CA3 neurons using the single-electrode voltage-clamp technique. The neuronal membrane potential was clamped to a holding potential $\left(\mathrm{V}_{\mathrm{H}}\right)$ of $-70 \mathrm{mV}$. Depolarizing voltage steps (300-500 ms in duration) up to a command potential $\left(\mathrm{V}_{\mathrm{C}}\right)$ of $-30 \mathrm{mV}$ elicited outward current responses (Fig. 3A), the amplitude of which remained unaltered in the presence of cromakalim $(100 \mu \mathrm{mol} / \mathrm{l}$, Fig. $3 \mathrm{~A})$. In contrast, when hyperpolarizing voltage steps were applied, a small but significant, cromakalim-induced increase in the amplitude of the inward current responses was observed in all neurons tested ( $n=5$, Fig. 3B). In the presence of cromakalim, the $\mathrm{V} / \mathrm{I}$-curve of these neurons revealed an increase in slope conductance at membrane potentials negative to $\mathrm{V}_{\mathrm{H}}$ (Fig. 3C). The observation that cromakalim enhanced the magnitude of the neurons' inward rectification in hyperpolarizing direction is in agreement with the results obtained under current clamp conditions and confirms our conclusion that cromakalim activates a voltage-dependent, inwardly rectifying potassium conductance.

\section{Effects of potassium-channel blockers on the action of cromakalim}

We used several potassium channel blockers to characterize the cromakalim-activated potassium conductance. In these experiments, the action of cromakalim $(100 \mu \mathrm{mol} / \mathrm{l})$ on the membrane potential and on the hyperpolarizing part of the $\mathrm{I} / \mathrm{V}$-curve was determined in the absence and presence of TEA $(10-15 \mathrm{mmol} / \mathrm{l}), 4$-AP $(0.1 \mathrm{mmol} / 1)$, tolbutamide $(1 \mathrm{mmol} / \mathrm{l})$, intracellularly injected $\mathrm{CsCl}$, extracellularly applied $\mathrm{CsCl}(2 \mathrm{mmol} / \mathrm{l})$, and $\mathrm{BaCl}_{2}(0.1-0.5 \mathrm{mmol} / \mathrm{l})$. In all neurons tested, neither TEA $(n=3)$ nor 4-AP $(n=3)$ nor intracellularly injected $\mathrm{CsCl}(n=3)$ affected the cromakalim-evoked hyperpolarization or resistance decrease. Tolbutamide, a blocker of ATP-sensitive potassium channels in peripheral tissue (Trube et al. 1986), has been shown to antagonize the action of cromakalim in skeletal muscle (Spuler et al. 1989). Since ATP-sensitive potassium channels have been described in CNS neurons (Ashford et al. 1988), we tested the effects of tolbutamide on the action of cromakalim on hippocampal CA3 neurons. Tolbutamide $(1 \mathrm{mM})$ itself depolarized the membrane potential by $4.0 \pm 1.0 \mathrm{mV}$, and increased $\mathrm{R}_{\mathrm{N}}$ by $13.3 \pm 3.3 \mathrm{MOhm}$ $(n=3)$. However, the action of cromakalim on membrane potential and $\mathrm{R}_{\mathrm{N}}$ was not influenced by tolbutamide $(n=3)$.

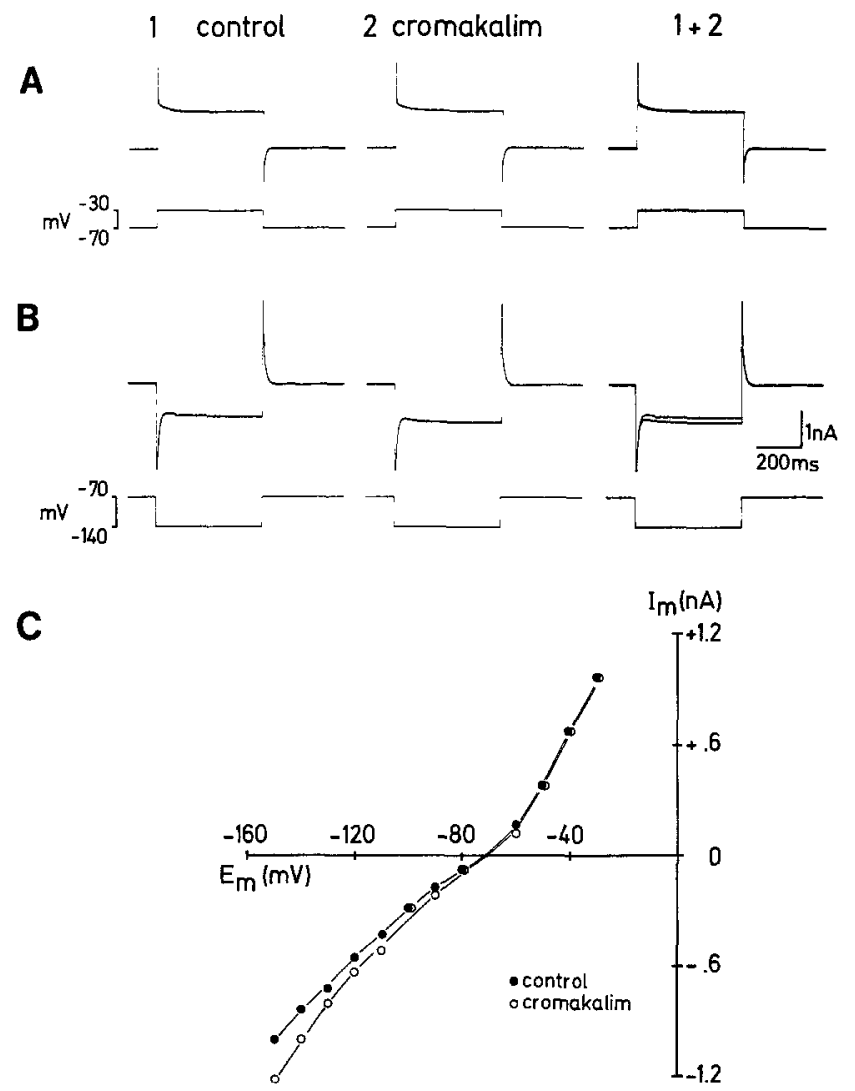

Fig. 3. Voltage-dependence of the action of cromakalim $(100 \mu \mathrm{mol} / 1)$. (A) In the presence of TTX $(0.6 \mu \mathrm{mol} / \mathrm{l})$ the membrane potential was voltage clamped to a holding potential of $-70 \mathrm{mV}$. A voltage step to a command potential of $-30 \mathrm{mV}$ (left panel, lower trace) produced an outward current response (left panel, upper trace) the amplitude of which did not change upon application of cromakalim (center traces). For a better demonstration, the right panel displays both measurements superimposed. (B) In the same neuron, a voltage step to a command potential of $-140 \mathrm{mV}$ (left traces) evoked an inward current response the amplitude of which increased upon application of cromakalim (center traces). The right traces depict both measurements superimposed. (C) Steady-state voltage-current curves determined in the absence (filled circles) and presence of cromakalim (open circles). The amplitudes of the current responses were plotted as a function of the attained command potentials. Holding potential was $-70 \mathrm{mV}$

Upon addition of $\mathrm{CsCl}(2 \mathrm{mmol} / \mathrm{l})$ to a TTX-containing bathing medium an increase in $\mathrm{R}_{\mathrm{N}}$ by $40-50 \%$ without changes in RMP was observed in all neurons tested $(n=5)$. Measurements of the I/V-relationship in a potential range negative to the RMP revealed a Cs ${ }^{+}$-induced block of the hyperpolarizing inward rectification (Fig. 4A). Under these conditions, cromakalim $(100 \mu \mathrm{mol} / 1)$ neither affected the membrane potential nor the I/V-relationship in 3 out of 5 neurons investigated (Fig. 4A). In the remaining two neurons, cromakalim produced membrane potential hyperpolarizations and decreases in $R_{N}$, the magnitudes of which were somewhat smaller but not significantly different from the cromakalim-induced changes in the absence of $\mathrm{Cs}^{+}$.

When added to a TTX-containing ACSF, $\mathrm{Ba}^{2+}$ led to an increase in $R_{N}$ by $80-100 \%$. This effect was accompanied by a slight depolarization of the membrane potential which we compensated for by hyperpolarizing DC-injection $(0.01-0.02 \mathrm{nA})$. Similar to $\mathrm{Cs}^{+}, \mathrm{Ba}^{2+}$ abolished or substan- 

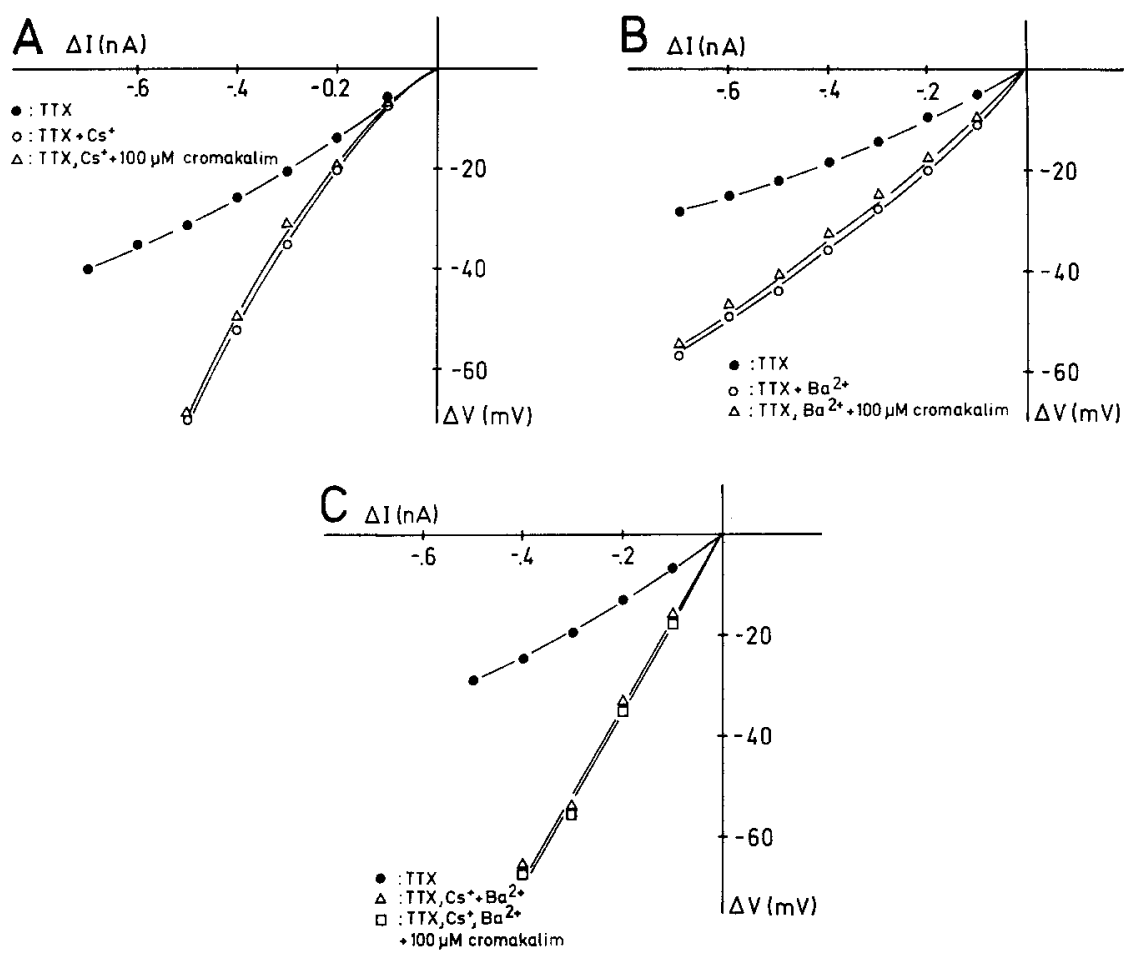

cromakalim

Control $100 \mu \mathrm{mol} / \mathrm{l}$

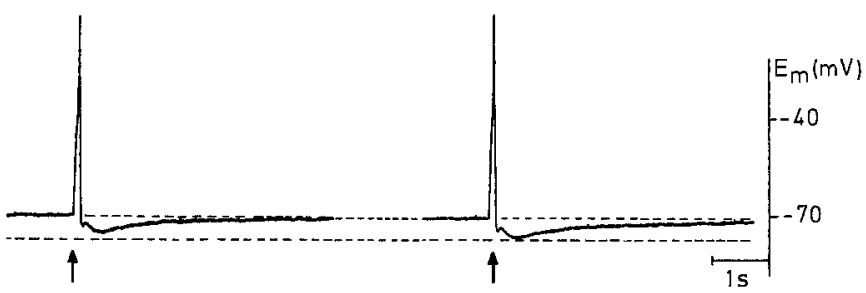

Fig. 5. Effect of cromakalim $(100 \mu \mathrm{mol} / \mathrm{l})$ on the slow after hyperpolarization following a single calcium-dependent action potential. In the presence of TTX $(0.6 \mu \mathrm{mol} / \mathrm{l})$ and $4-\mathrm{AP}(0.1 \mathrm{mmol} / \mathrm{l}) \mathrm{a}$ depolarizing current pulse (time of injection indicated by the arrow) evoked one calcium spike followed by a fast and slow afterhyperpolarization (control). The membrane potential was manually voltage-clamped to $-70 \mathrm{mV}$ (indicated by the upper dashed line). Upon application of cromakalim a slight increase in the amplitude and duration of the slow afterhyperpolarization could be observed. The lower dashed line indicates the maximal amplitude of the slow afterhyperpolarization in the presence of cromakalim

tially reduced the hyperpolarizing inward rectification and blocked the action of cromakalim on the membrane potential and on the hyperpolarizing I/V-relation in all neurons tested ( $n=4$, Fig. 4B). In two cells, a partial recovery of the $\mathrm{Ba}^{2+}$-induced changes was observed following a prolonged washout. Simultaneously, the effects of cromakalim were found to partially recover.

In 3 experiments, the slices were superfused with an ACSF containing TTX, $\mathrm{Ba}^{2+}$ and $\mathrm{Cs}^{+}$(Fig. 4C). Under these conditions, the hyperpolarizing I/V-relationship became steep and linear, and application of cromakalim was without any effect in all three neurons studied (Fig. 4C).

Recent experiments in peripheral tissues have provided evidence for a facilitatory effect of cromakalim on the TEA-
Fig. 4. Steady-state current-voltage-curves demonstrating the $\mathrm{Cs}^{+}$- and $\mathrm{Ba}^{2+}$-sensitivity of the action of cromakalim. Method of determination see Fig. 2. (A) The anomalous rectification seen under control conditions (i.e. in the presence of $0.6 \mu \mathrm{mol} / 1 \mathrm{TTX}$, closed circles) was blocked by $\mathrm{Cs}^{+}(2 \mathrm{mmol} / 1 \mathrm{CsCl}$, open circles) added to the bathing solution. Under these conditions, cromakalim $(100 \mu \mathrm{mol} / 1$, triangles) had no effect on the I/V-curve. (B) Similar measurement as in $\mathbf{A}$. Instead of $\mathrm{CsCl}$, $\mathrm{BaCl}_{2}(0.5 \mathrm{mmol} / 1)$ was added to the perfusion medium. (C) Lack of effect of cromakalim $(100 \mu \mathrm{mol} / 1$, squares $)$ in the presence of both $\mathrm{Cs}^{+}(2 \mathrm{mmol} / \mathrm{l})$ and $\mathrm{Ba}^{2+}$ $(0.5 \mathrm{mmol} / 1)$ insensitive, $\mathrm{Ca}^{2+}$-activated potassium conductance (Nakao et al. 1988). In central nervous system neurons, a similar potassium conductance has been demonstrated to be responsible for the slow afterhyperpolarization following a train of action potentials (Lancaster and Adams 1986). In order to investigate the effects of cromakalim on the $\mathrm{Ca}^{2+}$-activated potassium conductance, the slices were perfused with a medium containing both TTX and 4-AP. Under these conditions, the injection of a depolarizing current pulse evoked a single calcium-dependent action potential followed by an afterhyperpolarization with constant amplitude and duration (Fig. 5, control). Throughout the experiments, the membrane potential was manually voltage clamped to $-70 \mathrm{mV}$. Upon application of cromakalim $(100 \mu \mathrm{mol} / \mathrm{l})$, a slight, statistically marginally significant $(p<0.08)$, increase in amplitude and duration of the afterhyperpolarization was observed in all neurons tested ( $n=3$, Fig. 5), suggesting that, in addition to its facilitatory action on anomalous rectifying potassium conductances, cromakalim might have a weak potentiating effect on the TEA-insensitive $\mathrm{Ca}^{2+}$-activated potassium conductance in guinea-pig hippocampal CA3 neurons.

\section{Discussion}

The present results on the action of cromakalim on guineapig hippocampal CA 3 neurons obtained by means of intracellular recordings confirm and extend our observations made using extracellular recording techniques. As described in an earlier report (Alzheimer and ten Bruggencate 1988), cromakalim inhibited the spontaneous spiking activity of CA3 neurons. In the absence of TTX, this cromakaliminduced inhibition occurred without an apparent change in membrane potential (see Alzheimer and ten Bruggencate 1988). However, when spiking activity and spontaneous membrane potential oscillations were blocked by TTX, a 
cromakalim-induced hyperpolarization by $3-4 \mathrm{mV}$ associated with a decrease in $R_{N}$ was unmasked. As demonstrated in Fig. $1 \mathrm{~B}$ and $\mathrm{C}$, this small hyperpolarization per se is sufficient to account for the inhibitory effect of cromakalim on the spontaneous spiking activity of CA3 neurons. Furthermore, in contrast to membrane potential hyperpolarizations produced by DC injection, the cromakaliminduced hyperpolarization were accompanied by an increase in membrane conductance. Therefore, it can be assumed that the inhibitory efficacy of cromakalim-evoked hyperpolarizations is stronger than that of comparable hyperpolarizations caused by $\mathrm{DC}$ injections.

Although we did not perform a detailed analysis of the ionic mechanisms involved in the generation of the driving force for the cromakalim-induced hyperpolarization, our results strongly suggest that cromakalim hyperpolarizes the membrane of hippocampal CA 3 neurons by activation of a potassium conductance. Measurements of I/V-relationships before and during drug application revealed that the corresponding curves intercept at membrane potential values 20-30 $\mathrm{mV}$ negative to the RMP. Such potentials were close to the $\mathrm{E}_{\mathrm{K}}$ calculated for the given experimental conditions $(-96 \mathrm{mV}$ to $-102 \mathrm{mV}$ ). Furthermore, when the membrane potential was manually voltage-clamped to $-100 \mathrm{mV}$, cromakalim did not produce a hyperpolarization, but a slight depolarization of the membrane, indicating that the membrane potential was at, or slightly negative to, the equilibrium potential of the cromakalim-induced hyperpolarization. An involvement of chloride ions can be excluded, since all recordings were performed using electrodes filled with $\mathrm{KCl}$ or $\mathrm{CsCl}$. Leakage of chloride ions out of the electrodes led to a chloride equilibrium potential positive to the RMP, as revealed by depolarizing fast inhibitory postsynaptic potentials (data not shown).

In our first report on the action of cromakalim on CA3 neurons (Alzheimer and ten Bruggencate 1988), we observed that cromakalim was less effective at inhibiting spontaneous burst discharges in cells with RMP values slightly positive to the mean value of the total sample than in neurons with relatively high RMPs. This finding gave rise to the speculation that cromakalim might influence a voltage-dependent membrane conductance. The voltage-clamp experiments described in the present study show that cromakalim enhanced the membrane conductance at potentials negative to the mean RMP $(-69.5 \mathrm{mV}$, see Table 1$)$ without significantly affecting the conductance at potentials positive to the mean RMP (see Fig. 3). This is in agreement with the hypothesis that cromakalim activates or potentiates a voltage-dependent potassium conductance.

The investigation of the effects of well established blockers of potassium channels on the action of cromakalim provided further evidence that the compound influences the membrane properties by activation of voltage-dependent potassium conductances. While TEA, 4-AP, tolbutamide and internal $\mathrm{Cs}^{+}$did not antagonize the drug's action, the cromakalim-induced changes in membrane potential and $R_{N}$ were found to be blocked in the presence of $\mathrm{Cs}^{+}$and/or $\mathrm{Ba}^{2+}$. In different types of neurons, $\mathrm{Cs}^{+}$has been shown to abolish anomalous rectification (hyperpolarizing inward rectification) by inactivating a voltage-dependent, mixed sodium-potassium-conductance (for review see Adams and Galvan 1986). In addition, $\mathrm{Cs}^{+}$suppresses a fast activating inwardly rectifying potassium conductance demonstrated to be present in several types of central nervous system neurons
(Constanti and Galvan 1983; Stanfield et al. 1985; Williams et al. 1988). In contrast to $\mathrm{Cs}^{+}, \mathrm{Ba}^{2+}(100-500 \mu \mathrm{M})$ selectively blocks only the latter form of these inwardly rectifying conductances (Constanti and Galven 1983; Stanfield et al. 1985; Williams et al. 1988). Our observation that both $\mathrm{Cs}^{+}$and $\mathrm{Ba}^{2+}$ reduced or suppressed anomalous rectification indicates the presence of similar inwardly rectifying potassium conductances in guinea-pig hippocampal CA3 neurons. Furthermore, since cromakalim was without effect when anomalous rectification was virtually abolished by the simultaneous application of $\mathrm{Cs}^{+}$and $\mathrm{Ba}^{2+}$ (see Fig. $4 \mathrm{C}$ ), it is reasonable to conclude that cromakalim hyperpolarizes the membrane potential of hippocampal CA3 neurons by activation or potentiation of inwardly rectifying potassium conductances. An analogous action of cromakalim was described for guinea-pig isolated myocytes (Osterrieder 1988). In these cells, the drug activated a $\mathrm{Ba}^{2+}$ -sensitive, inwardly rectifying potassium conductance which resulted in a marked decrease in excitability. The concentrations necessary to evoke this effect $\left(10^{-5}-10^{-4} \mathrm{~mol} / \mathrm{l}\right)$ were similar to the concentrations necessary to induce inhibition in guinea-pig hippocampal CA3 neurons $\left(3 \times 10^{-5}-10^{-4} \mathrm{~mol} / \mathrm{l}\right)$.

In addition to producing a hyperpolarization and a decrease in $R_{N}$, cromakalim slightly enhanced the slow afterhyperpolarization following a single calcium-dependent action potential in hippocampal CA3 neurons (see Fig. 5). Since this afterhyperpolarization is most probably generated by the activation of a calcium-dependent potassium conductance (Lancaster and Adams 1986), we assume that cromakalim has a weak facilitatory effect on this conductance. This would be in agreement with observations made in smooth muscle cells, where the cromakalim-induced potassium-dependent hyperpolarization has been demonstrated to be sensitive to the calcium channel blockers $\mathrm{Mn}^{2+}$ and $\mathrm{La}^{3+}$ (Nakao et al. 1988).

In comparison to smooth muscle cells, where cromakalim at concentrations between 0.1 to $1 \mu \mathrm{mol} / 1$ causes almost maximal increases in potassium conductance and marked changes in ${ }^{86} \mathrm{Rb}^{+}$efflux (Cook 1988), cromakalim concentrations necessary to produce membrane hyperpolarizations, decreases in $R_{N}$, and reductions in epileptiform activity in hippocampal CA3 neurons are relatively high $(30-100 \mu \mathrm{mol} / 1)$. At the moment, there is no information about a cromakalim binding site or a "cromakalim-receptor". However, in order to produce its effect, cromakalim must bind either directly to the potassium channel protein or to another molecular structure which mediates the effect to the channel. The differences in the effective concentrations of cromakalim in smooth muscle preparations versus CNS preparations might be due to different binding sites with high and low affinity, or due to different effector systems, or both. The possibility that cromakalim acts as a weak agonist at a neurotransmitter receptor, the activation of which leads to an enhancement in anomalous rectification of CNS neurons, cannot be excluded. It has been demonstrated that 5-hydroxytryptamine (via the 5- $\mathrm{HT}_{1}$-receptor) and baclofen (via the GABA $\mathrm{B}_{\mathrm{B}}$-receptor) reduce the excitability of CNS neurons by the activation of an inwardly rectifying potassium conductance (Williams et al. 1988).

Acknowledgements. We would like to thank $\mathrm{L}$. Kargl for excellent technical assistance. This work was supported by the Deutsche 
Forschungsgemeinschaft (SFB 220) and by the Friedrich-BaurStiftung.

\section{References}

Adams PR, Galvan M (1986) Voltage-dependent currents of vertebrate neurons and their role in membrane excitability. In: Delgado-Escueta AV, Ward AA Jr, Woodbury DM, Porter RJ (eds) Advances in Neurology, Vol. 44. Raven Press, New York, pp $137-170$

Allen SL, Boyle JP, Cortijo J, Foster RW, Morgan GP, Small RC (1986) Electrical and mechanical effects of BRL 34915 in guineapig isolated trachealis. $\mathrm{Br} \mathrm{J}$ Pharmacol 89:395 - 405

Alzheimer C, ten Bruggencate G (1988) Actions of BRL 34915 (cromakalim) upon convulsive discharges in guinea pig hippocampal slices. Naunyn-Schmiedeberg's Arch Pharmacol 337: $429-434$

Ashford MLJ, Sturgess NC, Trout NJ, Gardner NJ, Hales CN (1988) Adenosine-5'-triphosphate-sensitive ion channels in neonatal rat cultured central neurones. Pflügers Arch 412:297304

Coldwell MC, Howlett DR (1987) Specificity of action of the novel antihypertensive agent, BRL 34915 , as a potassium channel activator. Comparison with nicorandil. Biochem Pharmacol $36: 3663-3669$

Connors BW, Gutnick MJ, Prince DA (1982) Electrophysiological properties of neocortical neurons in vitro. $J$ Neurophysiol $48: 1302-1320$

Constanti A, Galvan M (1983) Fast inward-rectifying current accounts for anomalous rectification in olfactory cortex neurones. J Physiol (Lond) 335:153-178

Cook NS (1988) The pharmacology of potassium channels and their therapeutic potential. TIPS 9:21-28

Gandolfo G, Gottesmann C, Bidard J-N, Lazdunski M (1989) $\mathrm{K}^{+}$ channels openers prevent epilepsy induced by the bee venom peptide MCD. Eur J Pharmacol 159:329 - 330

Ginsborg BL (1967) Ion movements in junctional transmission. Pharmacol Rev 19:289-316

Hablitz JJ, Johnston D (1981) Endogenous nature of spontaneous bursting in hippocampal pyramidal neurones. Cell Mol Neurobiol $1: 325-334$
Hamilton TC, Weir SW, Weston AH (1986) Comparison of the effects of BRL 34915 and verapamil on electrical and mechanical activity in rat portal vein. Br J Pharmacol 88:103-111

Johnston D, Hablitz JJ, Wilson WA (1980) Voltage clamp discloses slow inward current in hippocampal burst-firing neurones. Nature 286:391-393

Lancaster B, Adams PR (1986) Calcium-dependent current generating the afterhyperpolarization of hippocampal neurons. $J$ Neurophysiol 55:1268-1282

Nakao K, Okabe K, Kitamura K, Kuriyama H, Weston AH (1988) Characteristics of cromakalim-induced relaxations in the smooth muscle cells of guinea-pig mesenteric artery and vein. Br J Pharmacol 95:795-804

Osterrieder W (1988) Modification of $\mathrm{K}^{+}$conductance of heart cell membrane by BRL 34915. Naunyn-Schmiedeberg's Arch Pharmacol 337:93-97

Spuler A, Lehmann-Horn F, Grafe P (1989) Cromakalim (BRL 34915 ) restores in vitro the membrane potential of depolarized human skeletal muscle fibres. Naunyn-Schmiedeberg's Arch Pharmacol 339:327-331

Stanfield PR, Nakajima Y, Yamaguchi K (1985) Substance P raises neuronal membrane excitability by reducing inward rectification. Nature 315:498-501

Sutor B, Zieglgänsberger W (1987) A low-voltage activated, transient calcium current is responsible for the time-dependent depolarizing inward rectification of rat neocortical neurons in vitro. Pflügers Arch 410:102-111

Trube G, Rorsman P, Ohno-Shosaku T (1986) Opposite effects of tolbutamide and diazoxide on the ATP-dependent $\mathrm{K}^{+}$channel in mouse pancreatic $\beta$-cells. Pflügers Arch 407:493 - 499

Weir SW, Weston AH (1986) The effects of BRL 34915 and nicorandil on electrical and mechanical activity and on ${ }^{86} \mathrm{Rb}$ efflux in rat blood vessels. Br J Pharmacol 88:121-128

Williams JT, Colmers WF, Pan ZZ (1988) Voltage- and ligandactivated inwardly rectifying currents in dorsal raphe neurons in vitro. J Neurosci $8: 3499-3506$

Received April 25, 1989/Accepted July 14, 1989 